\title{
Trajectory Planning by Preserving Flexibility: Metrics and Analysis
}

\author{
Husni R. Idris ${ }^{*}$ and Tarek El-Wakil ${ }^{\dagger}$ \\ L3-Communications, Billerica, MA, 01821 \\ David J. Wing \\ NASA Langley Research Center, Hampton, VA, 23681
}

\begin{abstract}
In order to support traffic management functions, such as mitigating traffic complexity, ground and airborne systems may benefit from preserving or optimizing trajectory flexibility. To help support this hypothesis trajectory flexibility metrics have been defined in previous work to represent the trajectory robustness and adaptability to the risk of violating safety and traffic management constraints. In this paper these metrics are instantiated in the case of planning a trajectory with the heading degree of freedom. A metric estimation method is presented based on simplifying assumptions, namely discrete time and heading maneuvers. A case is analyzed to demonstrate the estimation method and its use in trajectory planning in a situation involving meeting a time constraint and avoiding loss of separation with nearby traffic. The case involves comparing path-stretch trajectories, in terms of adaptability and robustness along each, deduced from a map of estimated flexibility metrics over the solution space. The case demonstrated anecdotally that preserving flexibility may result in enhancing certain factors that contribute to traffic complexity, namely reducing proximity and confrontation.
\end{abstract}

\section{Nomenclature}

$\begin{array}{ll}\text { ADP } & \text { Adaptability } \\ \text { RBT } & \text { Robustness } \\ (\mathrm{t}, \mathrm{x}, \mathrm{y}) & \text { (time, } \mathrm{x} \text {-location, y-location) } \\ \mathrm{V} & \text { Ground speed } \\ \mathrm{h}_{\mathrm{i}}, \mathrm{h}_{\min }, \mathrm{h}_{\max } & \text { Heading with its maximum and minimum values } \\ \mathrm{Traj} & \text { Trajectory } \\ \mathrm{P}_{\mathrm{i}} & \text { Probability of trajectory instance traj } \mathrm{j}_{\mathrm{i}} \\ \mathrm{P}_{\mathrm{c}} & \text { Probability of constraint situation c } \\ \mathrm{P}_{\mathrm{f}} & \text { Probability of feasibility } \\ \mathrm{P}_{\mathrm{f}, \mathrm{c}} & \text { Probability of feasibility in constraint situation c } \\ \mathrm{f}(\mathrm{t}, \mathrm{x}, \mathrm{y}) & \text { Number of feasible trajectories from (t,x,y) to destination }\end{array}$

* Principal Research Engineer, 300 Concord Road, Suite 400, AIAA Member

${ }^{\dagger}$ Software Engineer, 300 Concord Road, Suite 400, AIAA Member

* Principal ATM Research Engineer, Mail Stop 152 


$\begin{array}{ll}\mathrm{f}_{\mathrm{c}}(\mathrm{t}, \mathrm{x}, \mathrm{y}) & \text { Number of feasible trajectories from }(\mathrm{t}, \mathrm{x}, \mathrm{y}) \text { to destination in situation } \mathrm{c} \\ \mathrm{i}(\mathrm{t}, \mathrm{x}, \mathrm{y}) & \text { Number of infeasible trajectories from }(\mathrm{t}, \mathrm{x}, \mathrm{y}) \text { to destination } \\ \mathrm{i}_{\mathrm{c}}(\mathrm{t}, \mathrm{x}, \mathrm{y}) & \text { Number of infeasible trajectories from }(\mathrm{t}, \mathrm{x}, \mathrm{y}) \text { to destination in situation } \mathrm{c} \\ \mathrm{N}(\mathrm{t}, \mathrm{x}, \mathrm{y}) & \text { Number of all trajectories from }(\mathrm{t}, \mathrm{x}, \mathrm{y}) \text { to destination } \\ \mathrm{N}_{\mathrm{c}}(\mathrm{t}, \mathrm{x}, \mathrm{y}) & \text { Number of all trajectories from }(\mathrm{t}, \mathrm{x}, \mathrm{y}) \text { to destination in constraint situation c } \\ \mathrm{g}(\mathrm{x}, \mathrm{y}) \text { or } \mathrm{g}_{\mathrm{k}}(\mathrm{x}, \mathrm{y}) & \text { Number of trajectories from } \mathrm{k}=(\mathrm{t}, \mathrm{x}, \mathrm{y}) \text { to next time step } \\ \varepsilon & \text { Duration between time increments }\end{array}$

\section{Introduction}

$\mathrm{T}$ he Next Generation Air Transportation System (NextGen) is expected to receive up to three times the current traffic demand by the year 2025. ${ }^{1}$ In order to handle the expected increase in air traffic NextGen will introduce key transformations in Air Traffic Management (ATM), three examples of which are: Net-enabled information access, which substantially increases information sharing and awareness of system operations among users and service providers; performance-based services, which make access to National Airspace System (NAS) resources, such as runways and airspace volumes, dependent on aircraft equipage and capability; and aircraft trajectory-based operations, which will require aircraft to precisely follow customized four dimensional (4D) trajectories consisting of a specified path and along-path time conformance requirements, thus ensuring separation and optimizing traffic flow management over different time horizons. ${ }^{1}$ These capabilities enable a more optimal allocation of functions among the air traffic system agents, such as moving the ATM system towards a distributed architecture. ${ }^{2}$ The premise of the distributed architecture is to mitigate the air traffic service provider workload as a constraint against increasing airspace capacity, through pilot and airline participation. This has important implications on the new role of centralized control, taking on higher level supervisory functions such as monitoring and intervention, as opposed to lower level active control, thus enabling additional cost savings. Enabling these capacity and cost gains depends on the ability of distributed actions to achieve overall ATM objectives such as maintaining safety and efficiency at acceptable levels. Several related NextGen concepts are being investigated, for example, delegating to the pilot more authority over the aircraft trajectory for separation assurance ${ }^{3,4}$ and delegating more responsibility to airline operation centers for traffic flow management $t^{5,6}$.

Research on distributed ATM has focused on the investigation of sharing the primary function of separation assurance between pilots and controllers. Pilots are assisted in predicting and resolving loss of separation by cockpit automation, known generally as Airborne Separation Assistance Systems (ASAS). ${ }^{7,8}$ Other ASAS efforts include the Mediterranean Free Flight Program (MFFP) where early experiments showed positive results of self separation operations. ${ }^{9,10}$ Similarly, extensive research has concentrated on assisting ground-based controllers by automation such as the Center TRACON Automation System ${ }^{11}$ to maintain controller workload at an acceptable level. Research on distributed traffic flow management has been limited, where the TFM function remains largely centralized with incorporation of user preferences in the air traffic manager decisions, such as in the Collaborative Decision Making (CDM) programs ${ }^{11}$ with recent effort to increase the user TFM responsibilities ${ }^{6}$.

Without regulating traffic beyond the separation assurance time horizon, complex traffic situations may arise characterized by high rates of potential conflicts and lack of conflict free trajectory solutions. Many approaches have been documented to define and measure traffic complexity, mostly as a representation of controller workload from the centralized/human-control perspective. These metrics were mostly based on airspace geometry, for example, aircraft density, sector geometry, traffic mix and distribution, traffic flow structure, and mix of aircraft types and performance characteristics. ${ }^{12}$ Other efforts emphasized cognitive elements of complexity, in particular the controller use of structure such as standard flows, grouping of traffic, and merge points. ${ }^{13}$ Some metrics were proposed that are independent of the airspace structure and controller perspective. For example, Delahaye et al introduced complexity metrics based traffic flow pattern organization or disorder (topological entropy). ${ }^{14}$ Such complex traffic situations are difficult to control, either by ground-based controllers or by air-based pilots and airlines, and may result in compromising safety. Therefore, avoiding or preventing such situations is a prerequisite function needed to enable manageable separation assurance and safety.

In order to support traffic management functions, such as mitigating traffic complexity, ground and airborne systems may benefit from preserving or optimizing trajectory flexibility. Trajectory flexibility preservation enables 
an aircraft to plan its trajectory such that it preserves a requisite level of maneuvering flexibility in accommodating disturbances, caused for example by other traffic and weather activity. The concept hypothesizes that by each aircraft preserving its own trajectory flexibility, using an air-based or ground-based system, aggregate system objectives, such as maintaining acceptable traffic complexity are naturally achieved. To help support this hypothesis trajectory flexibility metrics have been defined in previous work to represent the trajectory robustness and adaptability to the risk of violating safety and traffic management constraints. ${ }^{15,16}$ It is also hypothesized that minimizing the constraints imposed on a trajectory, without jeopardizing the intended ATM objectives, increases its flexibility. These functions offer a trajectory-oriented approach to managing traffic complexity, by explicitly planning aircraft trajectories, from the ground or the air, such that their contribution to complexity is minimized. This is contrasted with airspace-oriented approaches that aim to ensure that airspace structure (such as sector size and route patterns) and traffic characteristics (such as aircraft density) are designed to dynamically limit traffic complexity.

In this paper these flexibility metrics are instantiated in the case of planning a trajectory with the heading degree of freedom. After a brief review of the concept in the second section, an analytical framework and a metric estimation method are presented in the third section based on simplifying assumptions, namely discrete time and heading maneuvers. Then, the forth section presents a case analysis to demonstrate the estimation technique and its use in trajectory planning in a situation involving meeting a time constraint and avoiding loss of separation with nearby traffic. The case involves comparing multiple path-stretch trajectories, in terms of adaptability and robustness along each, deduced from a map of the estimated flexibility metrics over the solution space. The case demonstrated anecdotally that preserving flexibility may result in enhancing certain factors that contribute to traffic complexity, namely reducing proximity and confrontation.

\section{Concept of Trajectory-Oriented Traffic Complexity Management}

This concept introduces two trajectory-oriented functions, trajectory flexibility preservation and trajectory constraint minimization, that complement the core separation assurance function. ${ }^{15,16}$ Trajectory flexibility preservation plans the trajectory in a manner that affords the aircraft sufficient flexibility in accommodating disturbances that stem, for example, from other traffic or from weather activity. Flexibility preservation complements separation assurance both within the conflict resolution horizon and outside it within an extended flexibility planning horizon. Within the conflict resolution horizon, flexibility is used to select from many conflict resolution solutions one that affords the aircraft more flexibility, for example to adapt to potential intruder traffic behavior. As discussed in Idris et al., 2007, although flexibility preservation does not explicitly coordinate between the aircraft, it assists each ownship in reducing the risk of conflict due to the potential behavior of the surrounding traffic, thus resulting in implicit coordination..$^{15}$

Beyond the conflict resolution horizon, the flexibility preservation function plans the aircraft trajectory to minimize its exposure to disturbances such as weather cells and dense traffic areas. It is hypothesized that trajectory flexibility preservation naturally produces traffic situations that are less complex than without its application. Figure 1 depicts an example involving aircraft maneuvering between convective weather cells. On the left side each

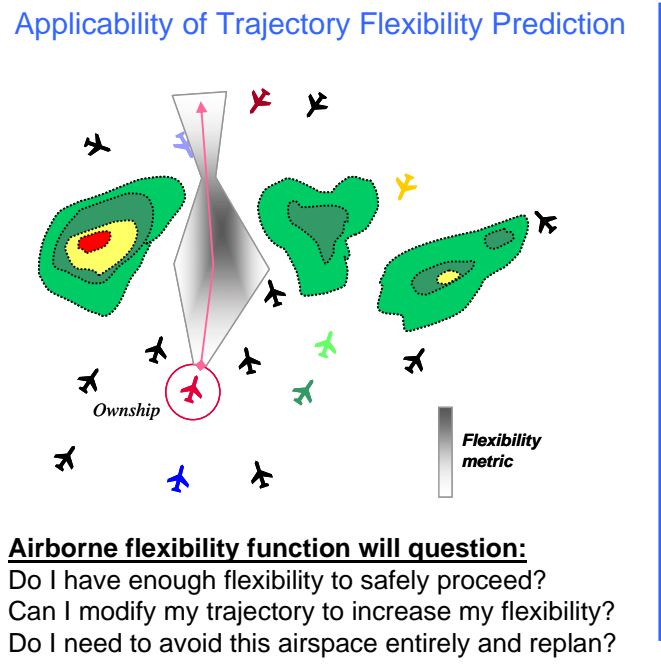

Trajectories Designed to Preserve Flexibility

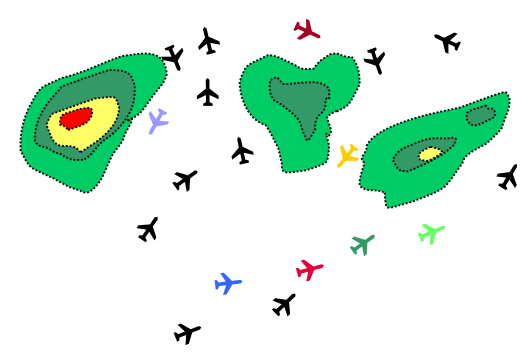

Hypothesis:

If all aircraft apply flexibility preservation function, complexity automatically will be reduced

Figure 1 Trajectory flexibility preservation avoiding weather cells and congestion 
aircraft, while planning its trajectory questions whether it should modify its trajectory to increase a flexibility metric. If the aircraft proceeded along their depicted headings a complex traffic situation arises causing excessive congestion and a high potential conflict rate in the airspace between the weather cells. On the other hand, the right side of the figure displays a more structured and streamlined traffic pattern that is hypothesized to result if each aircraft increased its flexibility - by limiting its exposure to the other traffic and the weather cells.

Trajectory constraint minimization assists a traffic manager to impose just enough constraints on the aircraft to meet an intended ATM objective. For example, if a single required time of arrival (RTA) at a specified fix will sufficiently meter the traffic flow, multiple RTAs per aircraft are deemed too excessive and hence candidate for relaxation. Trajectory constraint minimization enables more efficient utilization of NAS resources; but also increases the pilot's ability to maneuver freely with fewer constraints in order to accommodate disturbances. Therefore, the pilot may negotiate constraint reduction from the cockpit perspective. For example, the airborne automation may determine that the aircraft cannot meet all its constraints with enough flexibility, and hence the pilot may provide information to help the traffic manager relax some constraints. Figure 2 demonstrates the hypothesized impact of constraint minimization on trajectory flexibility preservation and hence traffic complexity. Ownship aircraft ' $A$ ' plans its trajectory to resolve a predicted conflict with aircraft ' $B$ ' within the conflict resolution lookahead and to meet an RTA at a downstream fix within the flexibility planning horizon. The RTA tolerance initially allows aircraft ' $A$ ' to avoid the predicted conflict only by path stretching to the left, with expected times of arrival (ETA) at the fix that lie within the RTA tolerance (left side of figure). These trajectories expose aircraft ' $\mathrm{A}$ ' to nearby traffic (Aircraft C and D) and an inclement weather system, and they would increase its contribution to traffic complexity. With this information, the traffic manager increases the RTA tolerance as shown in the right side of the figure, having determined that the intended ATM objectives can still be met sufficiently. With the extended tolerance, aircraft ' $\mathrm{A}$ ' has more maneuvering options to avoid the predicted conflict and select a more flexible trajectory with less exposure to the weather and traffic, thus reducing the aircraft contribution to traffic complexity. The aircraft is also able to meet its RTA constraint more accurately with less risk and hence achieve the intended ATM objectives more reliably.

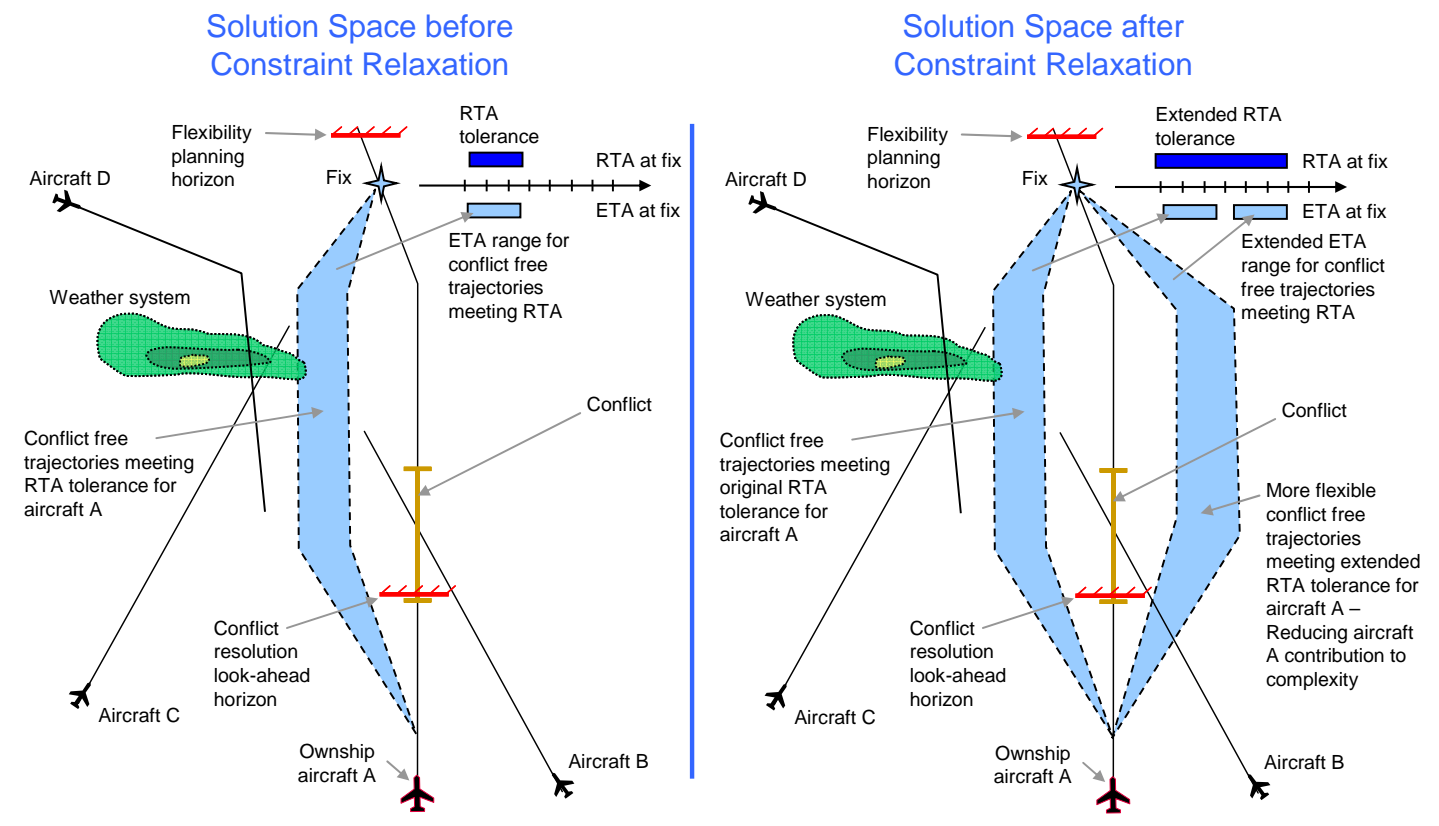

Figure 2. Trajectory constraint minimization example - relaxing RTA tolerance

\section{Analytical Framework and Metrics Estimation}

In order to define trajectory flexibility and to develop metrics and methods for trajectory flexibility preservation and constraint minimization, these notions and functions are posed in the framework of an aircraft trajectory solution space. The trajectory is required to abide by a set of constraints imposed to achieve ATM objectives such as maintaining separation requirements and balancing demand and capacity. Therefore, these constraints define a solution space consisting of the set of feasible trajectories. Out of these trajectories an aircraft selects one that 
optimizes its preferences, such as minimizing fuel burn, delay, and passenger discomfort. Here, the aim is to develop metrics that support selecting a trajectory that preserves flexibility and support identifying constraints that may be relaxed without jeopardizing the intended ATM objectives. A previous effort analyzed the solution space along a path using speed as the only degree of freedom and with RTA and conflict constraints. ${ }^{15,16,17}$ This analysis extends that research to the solution space for an aircraft with only heading as a degree of freedom and with RTA and conflict constraints.

\section{A. Trajectory Solution Space with RTA and Conflict Constraints}

In this paper the trajectory solution space is analyzed for an aircraft flying in a plane and with heading as the only degree of freedom. A number of assumptions are made in order to simplify the analysis, both for illustration of the concepts and tractability of metrics estimation. Figure 3 depicts in two dimensions $(\mathrm{x}, \mathrm{y})$ the trajectory solution space of an aircraft A flying towards a destination fix at $\left(\mathrm{x}_{\text {dest }}\right.$, $\left.\mathrm{y}_{\text {dest }}\right)$. Aircraft A may select a trajectory by selecting a heading profile $\mathrm{h}(\mathrm{x}, \mathrm{y})$ (several example are displayed in the figure) while keeping

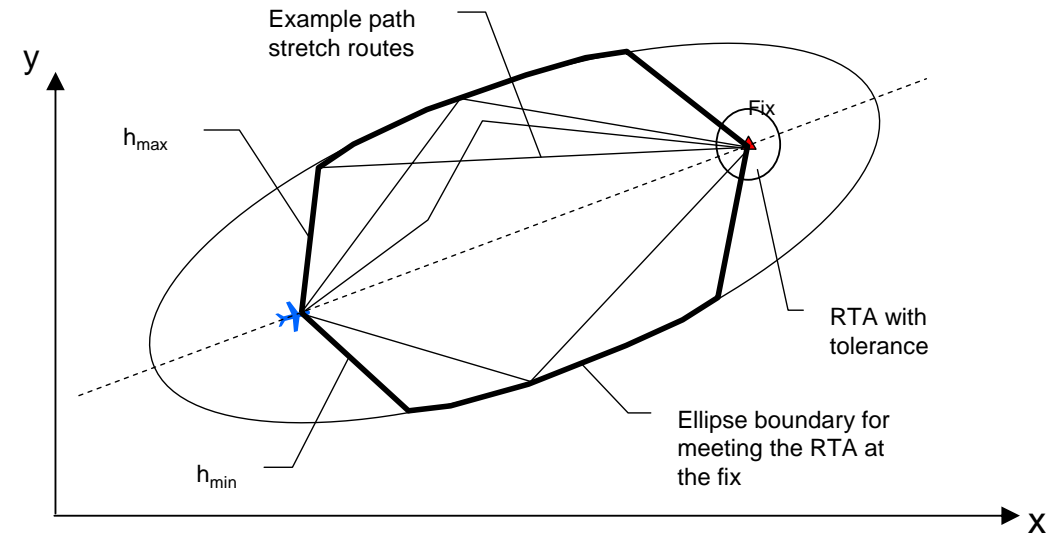

Figure 3. Solution space with RTA constraint its speed $\mathrm{v}(\mathrm{x}, \mathrm{y})$ along the path constant at a value $\mathrm{V}$. In this analysis, the aircraft trajectory is also constrained by maximum and minimum headings $\left(\mathrm{h}_{\max }\right.$ and $\mathrm{h}_{\min }$ respectively) at every point along the trajectory. These limits represent operational rather than physical constraints. They aim at confining the path stretch solution space to a reasonable area. For example, the heading bounds may be determined by a cone starting at the current position and surrounding the destination fix. ${ }^{\S}$ These heading limits also limit converging at the destination fix within a cone.

Aircraft A faces a constraint to meet an RTA at the destination fix. The RTA constraint reduces the reachable set by eliminating non-feasible regions, which consist of the reachable states that, if reached, the allowable heading range is not effective in meeting the RTA. In order to meet the RTA constraint at the destination fix, the path stretch solution space is limited to within an ellipse with focal points at the current and destination positions, as shown in Figure 3 for the fix point as the destination. The ellipse boundary results from the requirement that the trajectory length $\mathrm{D}$ is constant and equal to ((RTA - current time) times V). It corresponds to the trajectories that are anchored at the current and the destination positions and fully stretched outwards, resulting in a single heading change at the ellipse boundary. All other trajectories with length D and more than one heading change are stretched less and lie within the ellipse. If the destination allows a tolerance around it as shown in the figure, one such elliptical boundary corresponds to each allowable destination point within the tolerance. The path stretch solution space is then bound by the outmost one. Also, if the speed is allowed to vary between a minimum and a maximum value, the ellipse boundary corresponds to the longer distance required at the maximum speed.

\footnotetext{
$\S$ In general these bounds are function of the position along the trajectory and the previous heading used to get to that position (to avoid large heading changes). However, in this example, $\mathrm{h}_{\min }$ and $\mathrm{h}_{\max }$ are considered absolute and constant along the trajectory, for simplicity.
} 
While the elliptical boundary is an outer bound of the solution space, points within it may or may not be feasible depending on other constraints and operational assumptions. In this paper the aircraft is assumed to follow segments of discrete time length, with constant heading and speed along each segment. The aircraft is assumed to make heading changes at specific instances in time separated by a time increment during which it is maintained constant. As discussed later, this assumption simplifies a method for estimating the metrics proposed in Idris et al. ${ }^{17}$ This discretization method is demonstrated more explicitly by depicting the solution space in Figure 4 in three dimensions, $\mathrm{x}, \mathrm{y}$ and time t. Because of the constant speed assumption, the solution space lies on a series of conical shells; each corresponds to the allowable heading range and makes a slope with the time axis equal to the constant speed V of the aircraft. As shown in the figure, the first conical shell starts at the current position. ${ }^{* *}$ Then, the solution space remains on the conical shell as long as a selected heading is maintained constant, and a new cone originates from the point a new heading is selected. The new cone can be at the same or a new speed from the previous cone (but it assumed to be the same speed in this paper).

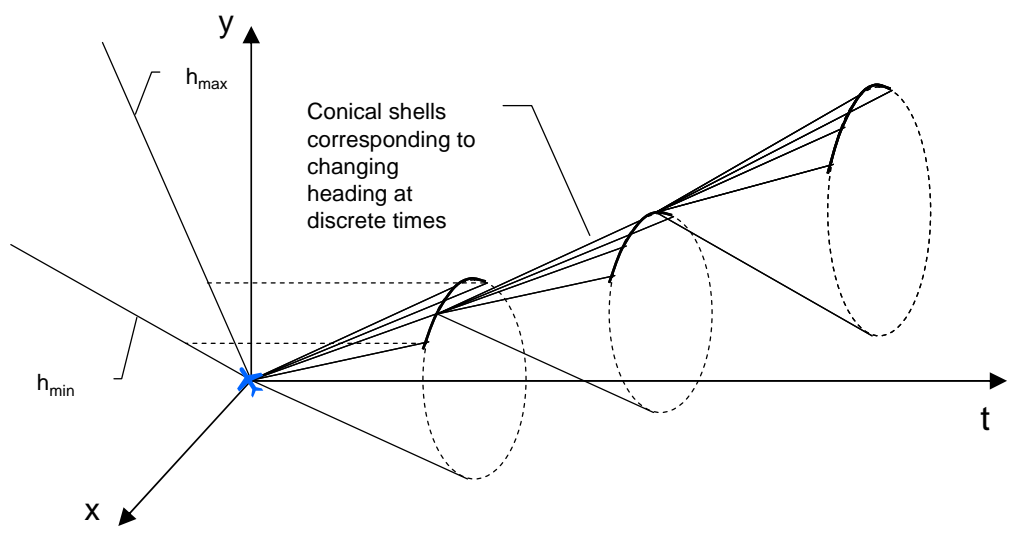

Figure 4. Discretization of the solution space

In addition to the RTA constraint aircraft A has to maintain separation from an intruder aircraft B (which may also represent in general a moving weather cell). This is depicted in Figure 5 by encircling the intruder aircraft with a circle of a radius equal to the minimum separation requirement (typically $5 \mathrm{nmi}$ ). The separation zone around the intruder aircraft becomes in the three dimensional space ( $\mathrm{t}, \mathrm{x}, \mathrm{y})$ a cylinder along its motion line, which is assumed to be along a constant speed and heading in this example. For illustration, the impact of the separation zone on the solution space is depicted in Figure 5 for a single conical shell extending from the current state for the duration of the RTA. Because of its maximum and minimum heading constraints, aircraft $\mathrm{A}$ has to remain outside a parallelepiped volume tangentially surrounding aircraft B's cylindrical separation zone. This volume is bound by four planes: each is tangent to the cylindrical separation zone and intersects the $x-y$ plane with a line of slope $\mathrm{h}_{\min }$ or $\mathrm{h}_{\max }$. A trajectory that crosses this region loses separation with the intruder aircraft and is therefore infeasible. A trajectory (which corresponds to a heading profile in this case) is feasible if it lies entirely in feasible regions. Therefore, for the aircraft

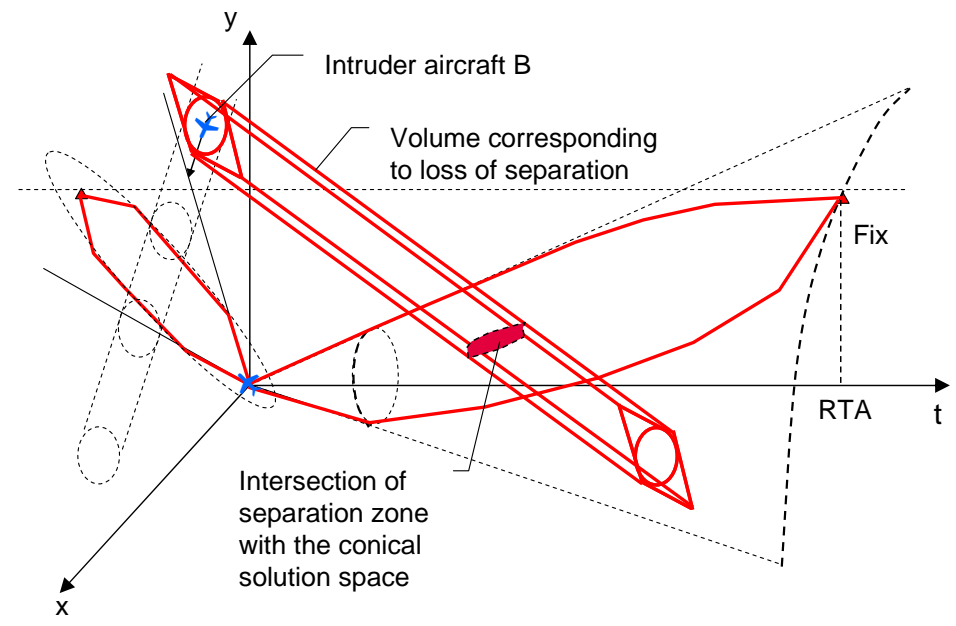

Figure 5. Solution space with RTA and conflict constraints to meet the RTA and be conflict free its trajectory has to lie with the elliptical boundary of Figure 4 and remain outside the parallelepiped volume around aircraft B.

Imposing more constraints further limits the trajectory solution space of the aircraft. The locations and tolerances of RTAs or the conflict regions may leave no feasible trajectory that is conflict free and meets constraints. In this case the aircraft trajectory is said to be over-constrained and requires relaxation of some constraints. Figure 3 demonstrates how relaxing a constraint by, for example, increasing the RTA tolerance opens up solution space and

** If the speed is allowed to vary between a maximum and a minimum speed the solution space lies within a conical volume bounded by a two conical shells, one corresponding to the minimum speed and one to the maximum speed. 
allows more feasible trajectories. A trajectory is said to be excessively constrained if the constraints allow some feasible trajectories but without sufficient flexibility, which is defined next.

\section{B. Trajectory Flexibility Metrics}

The notion of "trajectory flexibility" was defined in Idris 2007 as the ability of the trajectory (and hence the aircraft following the trajectory) to abide by all constraints imposed on it while mitigating its exposure to risks that cause violation of these constraints. ${ }^{15,16}$ Examples of these constraints include the heading limits, RTAs, and loss of separation, described in the previous section, but in general they include any constraints that intend to achieve ATM and aircraft objectives. They define the trajectory solution space as was shown in Figure 5.

Risk of constraint violation is represented by disturbances that alter the images depicted in Figure 5 causing the aircraft trajectory to violate or potentially violate constraints. Disturbances were classified in Idris 2007 into state or constraint disturbances. ${ }^{16}$ State disturbances result in aircraft state deviations along its trajectory. For example, the aircraft may pass through a turbulence region with uncertain wind speed, which results in the aircraft assuming one of many possible ground speeds in this region, some of which may lead to constraint violation. Constraint disturbances result in deviations in the constraints that define the aircraft trajectory solution space. They may be new constraints or modifications of currently imposed or known potential constraints. They include many types such as new TFM restrictions or new potential conflicts with traffic or weather cells, of which limited or no information may be available at the prediction time.

Two trajectory characteristics relevant to measuring this notion of flexibility have been identified: robustness and adaptability. ${ }^{15,16}$ Metrics have also been proposed in Idris 2008 for robustness and adaptability based on estimating the number of feasible trajectories available to the aircraft to accommodate disturbances. ${ }^{17}$

(1) Robustness is defined as the ability of the aircraft to keep its planned trajectory unchanged in response to the occurrence of disturbance. A trajectory that can withstand a disturbance without having to be changed is more robust than other trajectories that become infeasible when the disturbance occurs. In the context of the RTA/conflict constraint scenario of Figure 5, a trajectory that remains feasible in terms of meeting the RTA and avoiding the potential conflicts despite the disturbances shown in the figure (i.e., no matter which trajectory or conflict instances materialize) is robust to these disturbances.

A robustness metric RBT(traj) is associated with a trajectory (traj) starting from a state ( $t, x, y)$ and ending at another state such as $\left(\mathrm{RTA}, \mathrm{x}_{\text {dest }}, \mathrm{y}_{\text {dest }}\right)$. RBT(traj) is measured with the probability of feasibility $\mathrm{P}_{\mathrm{f}}(\operatorname{traj})$ of the trajectory, which can be estimated with partial information about state and constraint disturbances that represent the risk of constraint violation or infeasibility. Estimating $\mathrm{P}_{\mathrm{f}}($ traj) requires probabilistic models of the state and constraint disturbances. As an example, consider a state disturbance that makes every trajectory from any state ( $\mathrm{t}, \mathrm{x}$, $y)$ to the destination e.g., (RTA, $x_{\text {dest }}, y_{\text {dest }}$ ) possible with equal probability. In this case, with $\mathrm{N}(\mathrm{t}, \mathrm{x}, \mathrm{y})$ the total number of trajectory instances that start at state $(\mathrm{t}, \mathrm{x}, \mathrm{y})$ to the destination (in this case (RTA, $\mathrm{x}_{\text {dest }}, \mathrm{y}_{\text {dest }}$ )), the following formulas can be given for robustness RBT(t, $\mathrm{x}, \mathrm{y})$ See Idris 2008 for more detailed derivation: ${ }^{17}$

$$
P_{i}(t, x, y)=\frac{1}{N(t, x, y)} \quad, \quad P_{f, c}(t, x, y)=\frac{f_{c}(t, x, y)}{N(t, x, y)} \quad, \quad R B T(t, x, y)=P_{f}(t, x, y)=\sum_{c=l: C} P_{c} \times \frac{f_{c}(t, x, y)}{f_{c}(t, x, y)+i_{c}(t, x, y)},
$$

where the trajectory is modeled by $\mathrm{N}$ instances (traj $j_{i}$ ) each with probability $\mathrm{P}_{\mathrm{i}}$ with $\sum_{\mathrm{i}=1: \mathrm{N}} \mathrm{P}_{\mathrm{i}}=1$. The constraints are modeled with $\mathrm{C}$ constraint situations $\mathrm{c}$ each with a probability $\mathrm{P}_{\mathrm{c}}$ with $\sum_{\mathrm{c}=1 \mathrm{C}} \mathrm{P}_{\mathrm{c}}=1$. Each constraint situation $\mathrm{c}$ divides the total set of trajectories $N(t, x, y)$ into two mutually exclusive subsets: $f_{c}(t, x, y)$ the set of feasible trajectories with respect to $c$ and $i_{c}(t, x, y)$ the set of infeasible trajectories with respect to $c$. Hence, $N(t, x, y)=f_{c}(t, x, y)+i_{c}(t$, $\mathrm{x}, \mathrm{y})$.

(2) Adaptability is defined as the ability of the aircraft to change its planned trajectory in response to the occurrence of disturbance that renders the current planned trajectory infeasible. In the context of the RTA/conflict scenario of Figure 5, a trajectory that positions the aircraft such that other feasible trajectories remain accessible to it if a disturbance occurred and rendered the current planned trajectory infeasible is more adaptable than another trajectory for which the disturbance leaves fewer or no feasible trajectories.

An adaptability metric $\operatorname{ADP}(t, x, y)$ is associated with a state $(t, x, y)$ along a trajectory and is measured by the number of feasible trajectories $\mathrm{f}(\mathrm{t}, \mathrm{x}, \mathrm{y})$ (with respect to all constraints) that are available for the aircraft to use at (t, $\mathrm{x}, \mathrm{y})$ to regain feasibility. Then, given the probability distribution $\left(\mathrm{P}_{\mathrm{c}}\right)$ of the constraint situations $\mathrm{c}$ of $\mathrm{C}$ :

$$
\operatorname{ADP}(t, x, y)=f(t, x, y)=\sum_{c=1: C} P_{c} \times f_{c}(t, x, y) .
$$


Adaptability decreases as the aircraft moves along a trajectory because the number of feasible trajectories decreases. In the special case of robustness (to totally random state disturbances) as the number of feasible trajectories decreases, the ratio of feasible trajectories to the total number of trajectories increases because the total number may decreases by infeasible as well as feasible trajectories.

\section{Estimation of Number of Feasible Trajectories}

The calculation of the adaptability and robustness metrics requires estimation of the number of total and feasible trajectories from a state ( $\mathrm{t}, \mathrm{x}, \mathrm{y})$ to the destination (location, time, or both). A method is described in this section that estimates this number under the two simplifying assumptions described above (see Figure 4):

(1) Heading change can only occur at specific discrete instances in time that are $\varepsilon$ apart. Within each time increment the heading is maintained constant.

(2) Heading can take only discrete values $h_{i}$ between $h_{\min }$ and $h_{\max }$.

Under these assumptions the number of trajectories may be estimated using a convolution and filtering technique. Figure 6 demonstrates this method for calculating the number of feasible trajectories $\mathrm{f}_{\mathrm{c}}(\mathrm{t}, \mathrm{x}, \mathrm{y})$ from any

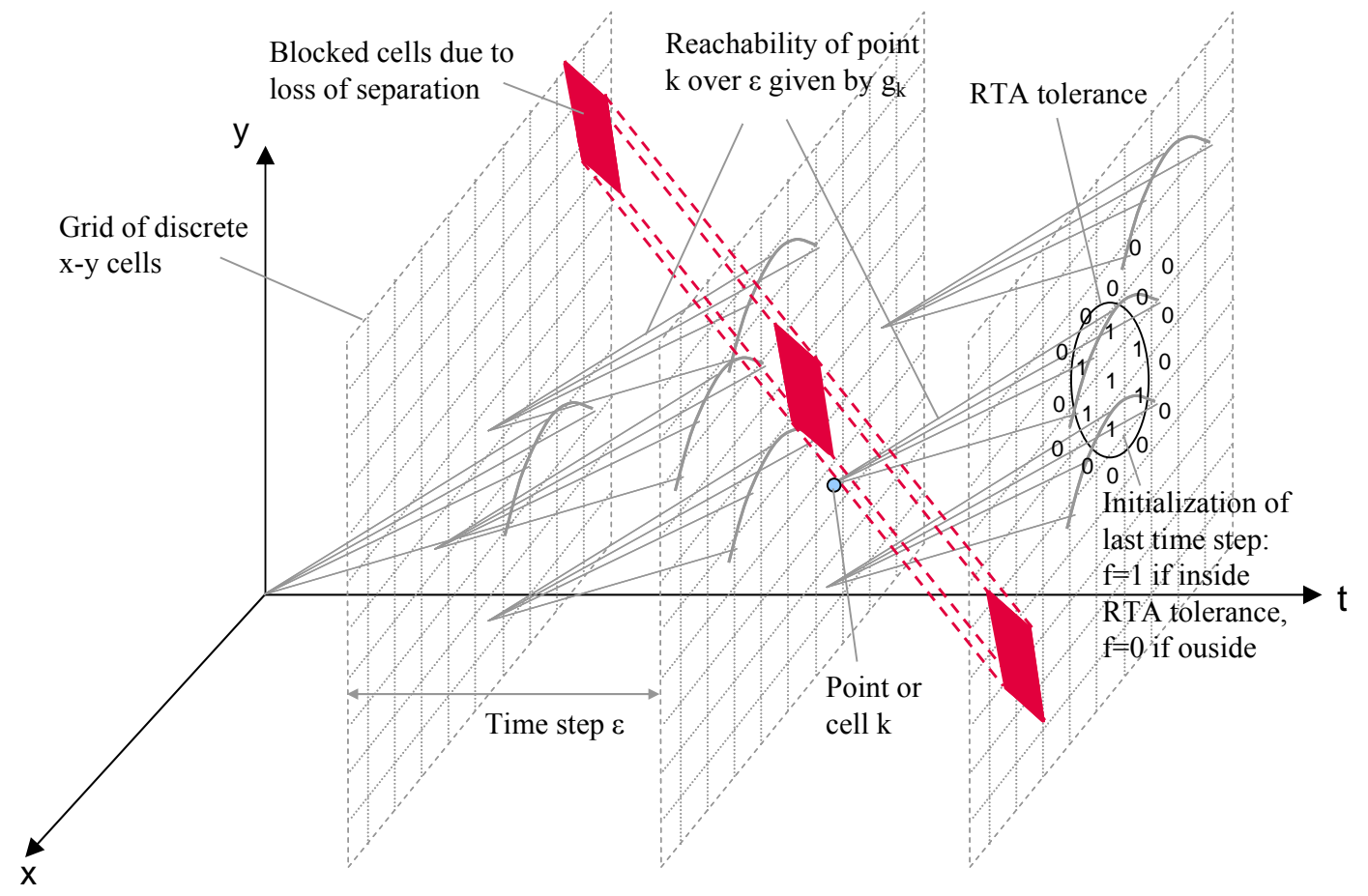

Figure 6. Estimation of number of feasible trajectories through convolution and filtering

point $(t, x, y)$ to a destination point (RTA, $x_{\text {dest }}, y_{\text {dest }}$ ) (with destination tolerance in $\mathrm{x}-\mathrm{y}$ ) in a constraint situation $\mathrm{c}$ that includes an instance of a potential conflict. The 3-dimensional space is discretized into time steps $\varepsilon$-apart, where in each time step the $x-y$ plane is discretized into square cells. The function $f_{c}(t, x, y)$ is estimated for each cell. Assume the function $f_{c}\left(t_{j}, x, y\right)$ at time $t=t_{j}$ is known. The function $f_{c}\left(t_{j-1}, x, y\right)$ at the previous time step $t=t_{j-1}$ can be obtained by convoluting $\mathrm{f}_{\mathrm{c}}\left(\mathrm{t}_{\mathrm{j}}, \mathrm{x}, \mathrm{y}\right)$ and the function $\mathrm{g}_{\mathrm{k}}(\mathrm{x}, \mathrm{y})$, which represents the number of trajectories that reach from a point $k=\left(t_{j-1}, x(k), y(k)\right)$ at time step $t_{j-1}$ to the next time step $t_{j}$. The function $g$ is independent of time because of the discretization assumptions Because of the assumptions of discrete heading values and constant heading between two time steps, there is only one trajectory that reaches from the point $k$ at time step $t_{j-1}$ to each of a set of discrete locations at time step $t_{j}$ - each location corresponds to one of the allowable discrete heading values $h_{i}$ between $h_{\min }$ and $h_{\max }$. Therefore, the reachability function $g_{k}(x, y)=1$ (i.e., one trajectory) at these discrete locations belonging to the set:

$$
\begin{aligned}
\left(\mathrm{t}_{\mathrm{j}}, \mathrm{x}, \mathrm{y}\right) \in\left\{\left(\mathrm{t}_{\mathrm{j}}, \mathrm{x}(\mathrm{k})+\mathrm{V} \times \cos \left(\mathrm{h}_{\text {min }}\right) \times \varepsilon, \mathrm{y}(\mathrm{k})+\mathrm{V} \times \sin \left(\mathrm{h}_{\min }\right) \times \varepsilon\right), \cdots,\right. \\
\left(\mathrm{t}_{\mathrm{j}}, \mathrm{x}(\mathrm{k})+\mathrm{V} \times \cos \left(\mathrm{h}_{\mathrm{i}}\right) \times \varepsilon, \mathrm{y}(\mathrm{k})+\mathrm{V} \times \sin \left(\mathrm{h}_{\mathrm{i}}\right) \times \varepsilon\right), \cdots, \\
\left.\left(\mathrm{t}_{\mathrm{j}}, \mathrm{x}(\mathrm{k})+\mathrm{V} \times \cos \left(\mathrm{h}_{\text {max }}\right) \times \varepsilon, \mathrm{y}(\mathrm{k})+\mathrm{V} \times \sin \left(\mathrm{h}_{\text {max }}\right) \times \varepsilon\right)\right\}
\end{aligned}
$$

and $g_{k}(x, y)=0$ elsewhere. The convolution operation amounts to calculating $f_{c}\left(t_{j-1}, x(k), y(k)\right)$ at point $k$, by multiplying the values of $f_{c}\left(t_{j}, x, y\right)$ by the number of trajectories that reach from point $k$ to $\left(t_{j}, x, y\right)$ and adding 
them, and then repeating the operation for each point $k$ in the $x-y$ plane at time step $t_{j-1}$. Therefore calculating $f_{c}\left(t_{j-1}\right.$, $x(k), y(k))$ at every point $k$ in the $x-y$ plane at time step $t_{j-1}$ amounts to adding the values of the function $f_{c}\left(t_{j}, x, y\right)$ that overlap the non-zero part of the function $\mathrm{g}_{\mathrm{k}}(\mathrm{x}, \mathrm{y})$. However, if the point $\mathrm{k}$ is infeasible (for example due to falling within the conflict zone of the solution space) then $\mathrm{f}_{\mathrm{c}}\left(\mathrm{t}_{\mathrm{j}-1}, \mathrm{x}(\mathrm{k}), \mathrm{y}(\mathrm{k})\right)=0$. This requires a filtering step after each convolution operation to zero out the values at infeasible states. Substituting a dummy variable $\tau$ to denote sliding the point $\mathrm{k}$ in the $\mathrm{x}-\mathrm{y}$ plane, the function $\mathrm{f}_{\mathrm{c}}\left(\mathrm{t}_{\mathrm{j}-1}, \mathrm{x}, \mathrm{y}\right)$ is given by the following equation, representing convolution and filtering for infeasibility:

$$
\begin{array}{ll}
\mathrm{f}_{\mathrm{c}}\left(\mathrm{t}_{\mathrm{j}-1}, \mathrm{x}, \mathrm{y}\right)=\sum_{\lambda} \sum_{\tau} \mathrm{f}_{\mathrm{c}}\left(\mathrm{t}_{\mathrm{j}}, \tau, \lambda\right) \times \mathrm{g}(\mathrm{x}-\tau, \mathrm{y}-\lambda) & \text { if }\left(\mathrm{t}_{\mathrm{j}-1}, \mathrm{x}, \mathrm{y}\right) \text { is feasible } \\
\mathrm{f}_{\mathrm{c}}\left(\mathrm{t}_{\mathrm{j}-1}, \mathrm{x}, \mathrm{y}\right)=0 & \text { if }\left(\mathrm{t}_{\mathrm{j}-1}, \mathrm{x}, \mathrm{y}\right) \text { is infeasible }
\end{array}
$$

This operation is applied starting from the destination point (RTA, $\left.\mathrm{x}_{\text {dest }}, \mathrm{y}_{\text {dest }}\right)$ and proceeding backwards to the current state (or origin). This is possible because the function $\mathrm{f}_{\mathrm{c}}(\mathrm{RTA}-\varepsilon, \mathrm{x}, \mathrm{y}$ ) (at the time step before last) is known due to the discrete speed assumption. This initialization may be achieved by setting at the final time step $t=R T A$, $\mathrm{f}_{\mathrm{c}}(\mathrm{RTA}, \mathrm{x}, \mathrm{y})=1$ at the feasible states and zero elsewhere; and applying the convolution/filtering process to calculate $\mathrm{f}_{\mathrm{c}}(\mathrm{RTA}-\varepsilon, \mathrm{x}, \mathrm{y})$.

To compute the total number of trajectories $\mathrm{N}_{\mathrm{c}}(\mathrm{t}, \mathrm{x}, \mathrm{y})$ the convolution is applied without filtering by the infeasible regions due to the conflict. Then, the estimates $\mathrm{f}_{\mathrm{c}}(\mathrm{t}, \mathrm{x}, \mathrm{y})\left(\right.$ or $\left.\mathrm{N}_{\mathrm{c}}(\mathrm{t}, \mathrm{x}, \mathrm{y})\right)$ in each constraint situation $\mathrm{c}$ are averaged over all constraint situations $\mathrm{C}$ to obtain the adaptability or robustness metrics under probabilistic models of disturbances as described in the previous section.

The convolution operation produces an exponential growth of the number of feasible trajectories $f_{c}(t, x, y)$ backwards with time, where the highest number of trajectories is at the current state and it decreases with time towards the destination. This depicts the decrease of adaptability with time. The infeasible regions eliminate trajectories as the function $\mathrm{f}_{\mathrm{c}}(\mathrm{t}, \mathrm{x}, \mathrm{y})$ is zeroed at infeasible states in each step before proceeding to the previous step. This filtering produces troughs or valleys in the function $\mathrm{f}_{\mathrm{c}}(\mathrm{t}, \mathrm{x}, \mathrm{y})$ depicting the impact of constraints. The larger the impact of a constraint is the larger the resulting trajectory elimination.

This behavior is demonstrated in the example shown in Figure 7 which depicts an implementation of the estimation algorithm in MATLAB. This example consists of an aircraft with an RTA constraint to be at ( 8 min, 20 $\mathrm{nmi}, 18 \mathrm{nmi})$ relative to the current position $(0,0,18 \mathrm{nmi})$ with a tolerance of $5 \mathrm{nmi}$ around the destination. In addition the aircraft path is impacted by a potential conflict with an intruder aircraft moving across at a heading of

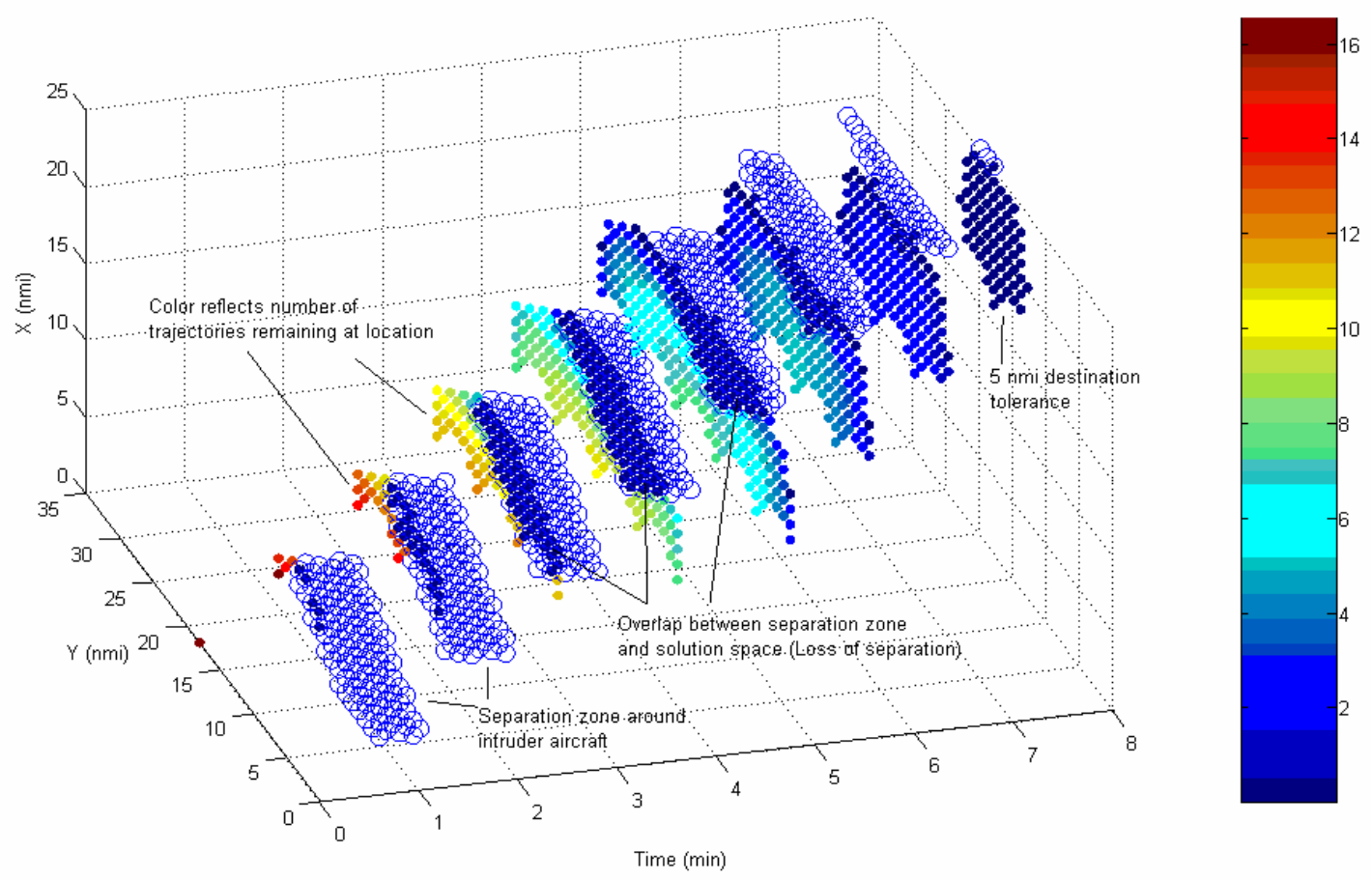

Figure 7. Trajectory number estimation example 
10 degrees from the right hand side of the aircraft. Both aircraft have a speed of 240 kts. Figure 7 shows in a t-x-y space the trajectory solution space of the aircraft with $h_{\min }$ and $h_{\max }$ set at -60 and +60 degrees, respectively. The intruder aircraft separation zone is shown by non-filled circles at each time steps. It also shows, as colored filled circles (the log value of) the number of feasible trajectories $f(t, x, y)$, remaining at each point, estimated with time increments of $1 \mathrm{~min}$ and heading increments of 20 degrees. Figure 7 shows the exponential decline of $f(t, x, y)$ from the origin towards the destination and the filtering out of trajectories in the troughs caused by the potential conflict region.

An identical convolution/filtering process may be applied in the reverse direction starting from the current state towards the destination, yielding the number of trajectories (total or feasible) that lead to each point (t, $\mathrm{x}, \mathrm{y}$ ). Denoting this number by $b(t, x, y)$, then for each point to be feasible there should be at least one trajectory to reach it from the current state and at least one trajectory to reach the destination from it. In other words the feasibility condition at a point $(t, x, y)$ may be written as: $f(t, x, y) \times b(t, x, y)>0$. These infeasible points are eliminated in Figure 7 showing the effect of discretization, where certain locations are unreachable from the current state due to the discrete heading and time assumptions.

\section{Analysis Case and Preliminary Insights}

A simple scenario is analyzed to demonstrate how the adaptability and robustness metrics described in the previous section can be used in trajectory flexibility planning. In this analysis the discretized metrics estimation over the solution space (of Figure 6) is used as a map to assess the adaptability and robustness of specific trajectories. Each trajectory is laid over the map and the number of trajectories that remain to the destination at each point along it is identified, as absolute value for adaptability and in relative terms for robustness. These numbers are then compared among different trajectories to select the optimal one based on adaptability, robustness, or a tradeoff between them. In this analysis the trajectories tested are generated independently of the map. Therefore, they do not have to abide by the discretization assumptions that were used to generate the map and may contain loss of separation. For example, a trajectory may have a heading that does not belong to the discrete set of headings used in the map and may change a heading at a time different than the discrete times used in the map for heading change. In these cases, the adaptability at a point along the trajectory is still measured by the number of trajectories remaining given the discretization assumptions from that point on, regardless of how the aircraft got to that point. This analysis is applied in the following scenario.

\section{A. Analysis Scenario}

The scenario analyzed, depicted in Figure 8, is a simplified representation of an aircraft (A) planning its trajectory between two streams of traffic, one along its direction and one in the opposite direction. The aircraft is constrained by a heading between -60 and +60 degrees relative to the line of sight between the current position $(\mathrm{x}=0, \mathrm{y}=18 \mathrm{nmi})$ and the destination fix position at $(\mathrm{x}=20, \mathrm{y}=18 \mathrm{nmi})$. It is also constrained to a constant speed of $240 \mathrm{kts}$. It has to meet an RTA at the destination fix at (8 $\mathrm{min}$ ) with a tolerance of $5 \mathrm{nmi}$ radius around the destination. Two intruder aircraft proceed on either side of the aircraft with a constant speed of 240 kts. Aircraft B starting at (x $=0$, $y=6 \mathrm{nmi}$ ) and at a heading of 0 degrees, along the same direction of aircraft $\mathrm{A}$, and aircraft $\mathrm{C}$ starting at $(\mathrm{x}=20 \mathrm{nmi}, \mathrm{y}=30 \mathrm{nmi})$ and with a heading of 180 degrees in the opposite direction to aircraft $\mathrm{A}$.

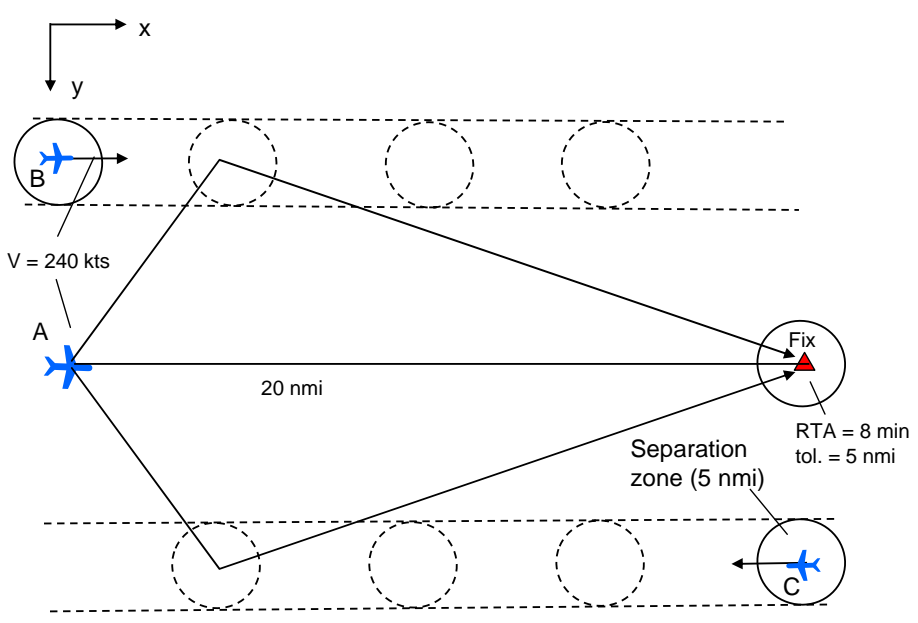

Figure 8. Analysis scenario

\section{B. Adaptability-Based Trajectory Planning}

The adaptability analysis of the scenario is shown in Figure 9, using the estimation method of the number of trajectories with 1-min time increments and 10-degree heading increments. A most adaptable heading profile would attempt to be as close as possible to the adaptability maxima at each time step, while ensuring reachability along the 
trajectory. Algorithms for finding such an optimum trajectory using the adaptability map are subject of future research. Preliminary insights are made in this analysis by showing in Figure 9 the optimal values with X symbols connected with a line. Also two single-turn trajectories are depicted, one starting at +60 degree heading and one starting with -60 degree heading and both ending at the destination fix. The following observations are made from Figure 9:
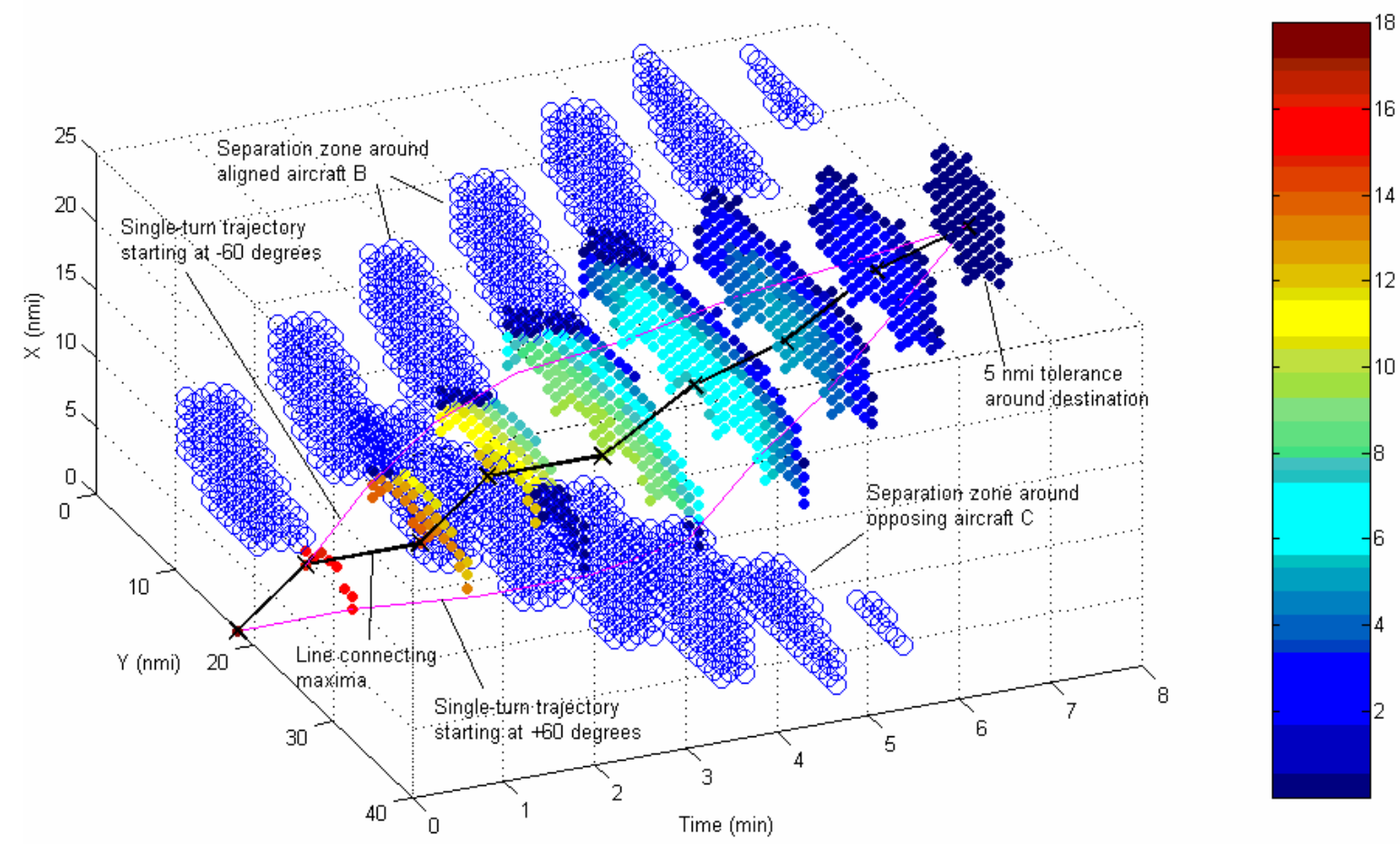

Figure 9. Adaptability analysis

(1) The opposing aircraft (C) impact is minimal in the time steps 8 to 4 where its separation zone does not overlap the solution space. Its impact is then concentrated at time step ' 3 ' where it its separation zone eliminates a large number of trajectories (shown as dark blue circles cluster). On the other hand, the aligned aircraft (B) impact is higher at the later time steps and is maintained throughout the time horizon where its separation zone overlaps the solution space in almost very time step. However, the cumulative filtering by the aligned aircraft, although spread over time, is less severe, resulting in higher number of trajectories remaining on the 'left' side of aircraft A than at the 'left' side.

(2) As a result of (1), the adaptability maxima indicate that the aircraft would optimize adaptability by flying first away from the opposing aircraft (C) and towards the aligned aircraft (B). Then after clearing the impact aircraft $\mathrm{C}$ it would apt to fly away from aircraft B increasing its separation from it. Finally it proceeds towards the destination using a central line where neither aircraft has an impact on the solution space (the central line is selected to break ties between many equally adaptable locations).

(3) If the aircraft is to optimize adaptability it has to make more than one turn (heading change). If it is limited to a single turn, for example, it would choose the one that start at -60 degrees, over the one that start with +60 degrees. In fact, the figure shows that the one starting with +60 degrees loses separation with aircraft $\mathrm{C}$.

\section{Robustness-Based Trajectory Planning}

Figure 10 repeats the analysis of Figure 9 based on maximizing robustness rather than adaptability, using the ratio of $f(t, x, y)$ to $N(t, x, y)$, thus assuming totally random state disturbance. For comparability with the adaptability analysis of Figure 9, in this example the total number of trajectories $\mathrm{N}(\mathrm{t}, \mathrm{x}, \mathrm{y})$ allowed by state (heading) variation is limited to the ones that meet the destination RTA constraint. This assumes that meeting the RTA constraint is totally robust to heading changes and robustness only with respect to meeting the conflict constraint is considered. In addition to the observations in the adaptability analysis the following observation are be made from Figure 10: 
(1) Robustness (in this special case) increases over time, as opposed to adaptability which decreases over time, reaching a maximum value of 100 percent near the destination.

(2) The robustness maxima indicate that the aircraft would optimize robustness also by flying first away from the opposing aircraft $(\mathrm{C})$ and towards the aligned aircraft $(\mathrm{B})$. Then after clearing the impact of aircraft $\mathrm{C}$ it would apt to fly away from aircraft B increasing its separation from it. Finally it proceeds towards the destination using a central line where neither aircraft has an impact on it (the central line is again selected by breaking ties between many equal options). This robustness result in this scenario is similar to the case of adaptability, which is not necessarily always the case.

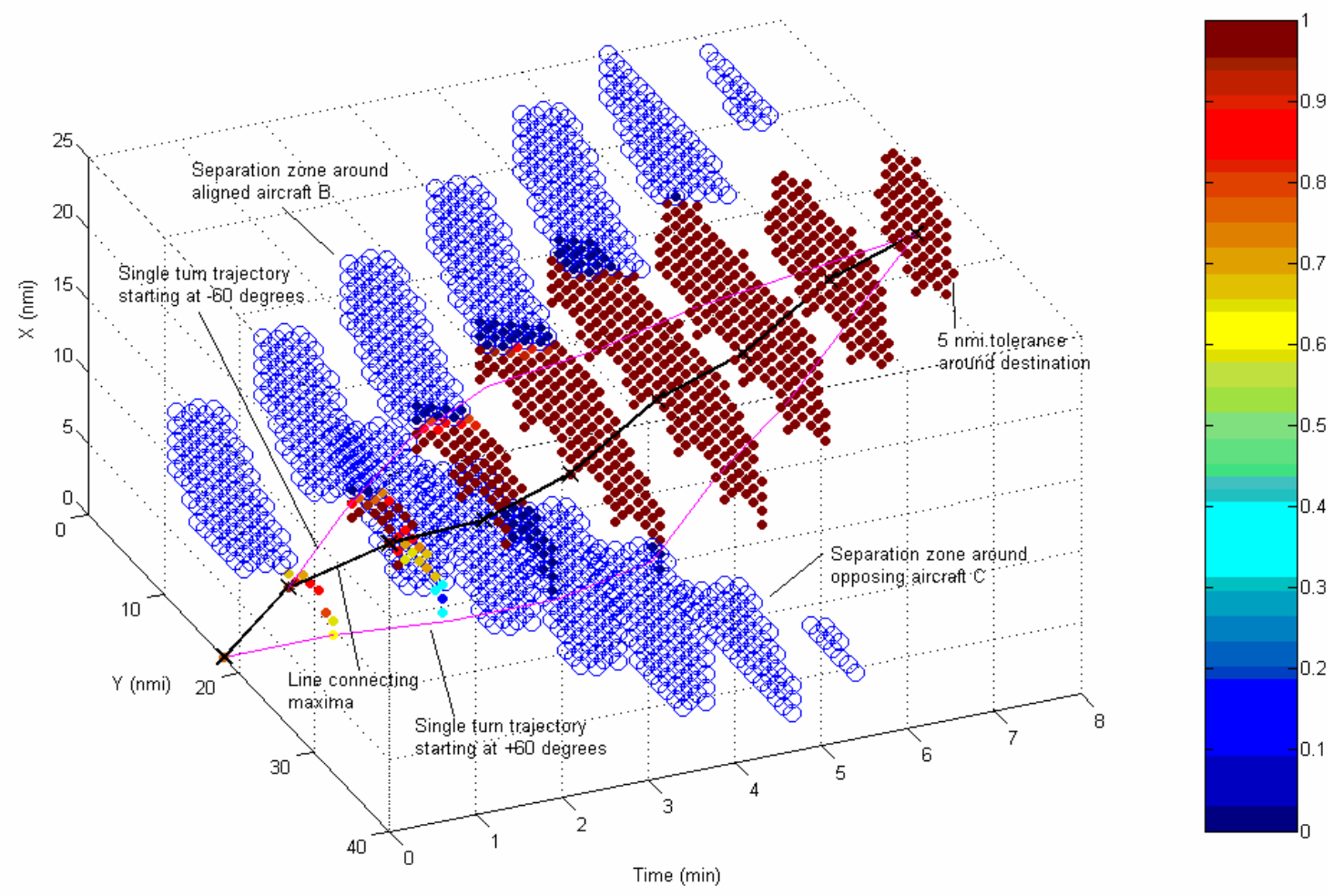

Figure 10. Robustness analysis

\section{Note on Constraint Minimization}

The adaptability and robustness metrics proposed can be used for assessing the impact of different constraints on the aircraft trajectory in terms of its flexibility. For example, the number of feasible trajectories $f(t, x, y)$ or its ratio to the total number of trajectories $\mathrm{N}(\mathrm{t}, \mathrm{x}, \mathrm{y})$ may be compared between situations involving different number and types of imposed constraints. Such comparison identifies relative gains from relaxing certain constraints in terms of flexibility. In turn, the relative gain from relaxing certain constraints (in terms of adaptability or robustness or a combination of both) can be used to support making decisions about constraint relaxation. Algorithms for using these metrics in making such decisions are a research topic that will be published in future papers.

\section{E. Note on Traffic Complexity Impact}

The metrics proposed in this paper will be ultimately used for trajectory flexibility planning and constraint minimization and analyzing the impact on traffic complexity. While these extensions are topics of future research, preliminary insights can be gained from this scenario. Traffic complexity involves a large number of factors. In this scenario, two factors may be identified as relevant, one related to the relative heading of the aircraft and one related to the proximity between them. The complexity of the traffic situation depends on how these factors trade, which is often subjective. However, the following observation may be made based on the most flexible trajectory (heading profile) selected by the aircraft. It is observed that the aircraft minimized the confrontation with the opposing traffic 
when its impact dominated (early), at the expense of closer proximity to the aligned traffic. Then when the opposing traffic was not a factor, it decided to reduce its proximity to the aligned traffic in later time steps. Although anecdotal, these observations give preliminary insight on the possible positive impact that may be expected on traffic complexity in more elaborated scenarios.

\section{Conclusions and Future Extensions}

In summary, a trajectory-oriented approach to managing traffic complexity was presented in this paper: Trajectory flexibility preservation enables an aircraft or a ground-based agent to preserve flexibility in accommodating disturbances from, for example, other traffic and weather activity. In addition, trajectory constraint minimization enables ground-based agents, in collaboration with air-based agents, to impose just-enough constraints on trajectories to achieve ATM objectives, such as separation assurance and flow management. The concept hypothesizes that by preserving trajectory flexibility, aggregate system objectives, such as maintaining acceptable traffic complexity, are naturally achieved. It also hypothesizes that minimizing the constraints imposed on a trajectory increases its flexibility. Towards testing these hypotheses, metrics associated with trajectory flexibility, defined in terms of robustness and adaptability to disturbances that present constraint violation risk, were derived in the case of the heading degree of freedom. A metric estimation method was developed under discretization assumptions of time and heading changes. The method was demonstrated and analyzed in the context of a simple scenario. The scenario involved the selection of path with only heading variation along the path, in simple constraint situations involving meeting one time of arrival and avoiding potential conflicts. The analysis of the scenario demonstrated the use of the robustness and adaptability metrics in trajectory planning, and the hypothesized relationship between trajectory flexibility preservation and traffic complexity management using relative heading and proximity as simple complexity indicators.

Future research plans include prototyping the metrics proposed in this paper in the AOP research model to support conducting experiments to test the hypothesized relationships and the proposed operational concepts. The metrics proposed in this paper will be prototyped in AOP, both to add flexibility preservation criteria to conflict resolution within the conflict resolution horizon, and to support a flexibility preservation function beyond the conflict resolution horizon.

To support such an experiment, a number of extensions to the work presented in this paper are planned. These extensions include generalizing the metrics and their estimation technique to situations involving other types and combinations of constraints and disturbances. This multiplicity of the constraints and their types also gives rise to a prioritization among them, which will be investigated in the context of the constraint minimization function. For example, when the aircraft is over-constrained it may report to the ground-based traffic manager that it is unable to meet an RTA because of a conflict. In this case the traffic manager may relax the RTA ensuring safety at the expense of TFM objectives.

Flexibility was defined in this paper as the aircraft ability to accommodate disturbances while abiding by all constraints. This goal is traded with other objectives of the aircraft such as fuel efficiency and other user preferences. Such tradeoffs will be investigated and may be incorporated in an overall trajectory planning function as objectives that compete with the flexibility preservation objective. Finally, the hypothesis made is that the flexibility preserving behavior of each individual aircraft will produce implicit coordination between aircraft and naturally result in less complex traffic situations. However, some level of explicit coordination may be needed to further mitigate traffic complexity. Future research will investigate, for example, the impact and need of information sharing between aircraft to coordinate the distributed flexibility preservation actions as well as the constraint minimization decisions.

\section{Acknowledgment}

This research was funded by NASA under contract NNA07BA86C.

\section{References}

${ }^{1}$ Joint Planning and Development Office, "Next Generation Air Transportation System Integrated Plan," URL: http://www.jpdo.gov/library/NGATS_v1_1204r.pdf

${ }^{2}$ Wing, D., "A Potentially Useful Role for Airborne Separation in 4D-Trajectory ATM Operations," Proceedings of the $5^{\text {th }}$ AIAA Aviation Technology Integration and Operations (ATIO) Conference, AIAA-2005-7336, 2005. 
${ }^{3}$ NASA, DAG-TM Concept Element 5 En Route Free Maneuvering for User-Preferred Separation Assurance and Local TFM Conformance Operational Concept Description, NASA Advanced Air Transportation Technologies Project Milestone 8.503.10, NASA Airspace Systems Program Office, Washington D.C., 2004.

${ }^{4}$ NASA, DAG-TM Concept Element 11 Terminal Arrival Self-Spacing for Merging and In-Trail Separation Operational Concept Description, NASA Advanced Air Transportation Technologies Project Milestone 8.652.7, NASA Airspace Systems Program Office, Washington D.C., 2004.

${ }^{5}$ Green, S. M., Bilimoria, K. D., and Ballin, M. G., "Distributed Air/Ground Traffic Management for En Route Flight Operations." Air Traffic Control Quarterly, Vol. 9, No. 4, 2001, pp. 259-285.

${ }^{6}$ Idris, H., Vivona, R., Penny, S., Krozel, J., and Bilimoria, K., "Operational Concept for Collaborative Traffic Flow Management based on Field Observations," Proceedings of the $5^{\text {th }}$ AIAA 5th Aviation Technology, Integration and Operations (ATIO) Conference, AIAA-2005-7434, 2005.

${ }^{7}$ Barhydt, R., and Kopardekar, P., "Joint NASA Ames/Langley Experimental Evaluation of Integrated Air/Ground Operations for En Route Free maneuvering," $6^{\text {th }}$ USA/Europe Air Traffic Management R\&D Seminar, 2005.

${ }^{8}$ Krishnamurthy, K., Barmore, B., and Bussink, F., "Airborne Precision Spacing in Merging terminal Arrival Routes," $6^{\text {th }}$ USA/Europe Air Traffic Management R\&D Seminar, 2005.

${ }^{9}$ Mediterranean Free Flight Programme Final Report, D821, http://www.medff.it/public/index.asp, November 2005.

${ }^{10}$ Blom H.A.P., B. Klein Obbink, and G.I. Bakker, "Safety Risk Simulation of an Airborne Self Separation Concept of Operation," Proceedings of the $7^{\text {th }}$ AIAA Aviation Technology Integration and Operations (ATIO) Conference, AIAA 2007-7729.

${ }^{11}$ Erzberger, H., T.J. Davis, and S.M. Green, "Design of Center-TRACON Automation System," AGARD Meeting on Machine Intelligence in ATM, Berlin, Germany, 1993

${ }^{12}$ Kopardekar. P. and S. Magyarits, "Measurements and prediction of dynamic density," $5^{\text {th }}$ USA/Europe ATM $R \& D$ Seminar, 2003

${ }^{13}$ Davison, J., Histon, J., Ragnarsdottir, M., Major, L., Hansman, R.J., "Impact of Operating Context on the Use of Structure in Air Traffic Controller Cognitive Processes". $5^{\text {th }}$ USA/Europe Air Traffic Management R\&D Seminar, 2002.

${ }^{14}$ Delahaye, D. Puechmorel, S., Hansman, R.J., and Histon, J., "Air traffic complexity based on non linear dynamical systems", $5^{\text {th }}$ USA/Europe Air Traffic Management R\&D Seminar, 2003.

${ }^{15}$ Idris H., D. Wing, R. Vivona, and J.L. Garcia-Chico, "A Distributed Trajectory-Oriented Approach to Managing Traffic Complexity," Proceedings of the $7^{\text {th }}$ AIAA Aviation Technology Integration and Operations (ATIO) Conference, AIAA-2007-7731, 2007.

${ }^{16}$ Idris H., R. Vivona, J.L. Garcia-Chico, and D. Wing, "Distributed Traffic Complexity Management by Preserving Trajectory Flexibility," Proceeding of the 26 ${ }^{\text {th }}$ Digital Avionics Systems Conference, 2007.

${ }^{17}$ Idris H., and R. Vivona, "Metrics for Traffic Complexity Management in Self-Separation Operations," Air Traffic Control Quarterly, (to be published). 